\title{
The increasing prevalence of cesarean in Iran: How the rate of cesareans could be controlled?
}

\section{Executive summary}

Pulling out the embryo, placenta and the membrane by cutting the abdomen and the womb is called cesarean and it is considered as a method of reduce maternal and neonatal mortality, but in past three decades there has been an increase in the rate of cesarean around the world which turned it into a public dilemma. The procedure of cesarean in Iran as well as developed and developing countries has been growing rapidly and its rate is much higher than the World Health Organization standards. In so many cases, the medical necessities do not result in a cesarean but it is due to the lack of knowledge, nontested and false beliefs, behaviors, and attitudes have determined the type of child delivery. ${ }^{1}$

Currently, 50-60 percent of the child deliveries are cesarean 90 percent of which is performed in cities and private hospitals. ${ }^{2}$ Cesarean engages so many of the hospital facilities, beds and experienced personnel while the rate of mortality and child delivery complications for mothers under this operation is remarkably high in comparison to the mothers who have natural child delivery.

Although Ministry of Health, Treatment and Medical Education has started a serious trial to reduce unnecessary cesarean since 2003, yet it has not achieved a considerable success in the context of reducing the growth of cesarean in the country. ${ }^{3}$ A comprehensive analysis of medical studies and scientific documents shows that designing an appropriate and evidence-based program for improving awareness of mothers regarding the advantages and disadvantages of the types of child delivery including cesarean and physiologic delivery (painless child delivery) has been an effective action in order to help them choose the best type of child delivery. Also by changing the behavior of service providers and monitoring them, an effective control on cesarean rate can be applied. ${ }^{4,5}$

\section{Statement of problem}

Child delivery is a natural process that should be done with the least of medical interferes ${ }^{6}$ and it is reasonable to apply cesarean as an strategy to save the lives of the mother and the child in difficult child deliveries, but in the last three decades, the rate of cesarean had a significant rise both in developed and developing countries which has caused it into a concern for experts and policymakers of health. ${ }^{4-9}$ This type of child delivery is considered as the most prevalent type of operation and also a common obstetric surgery all over the world. ${ }^{10}$ America and Australia with a percentage of 30/3 had the highest statistics among the developed countries in 2012, and some of developing countries such as Chile (40\%), Brazil, Korea, China (46\%), Iran, Pakistan, India and Turkey (25-40\%) have sounded bell. This is while based on the report of WHO, a maximum of $15 \%$ of the child deliveries have the indication of cesarean and a higher rate of it is unnecessary and inappropriate which inflict a financial burden and medical risk upon the patient and the system of health. ${ }^{12}$

Studies show that the economic burden and the burden of cesarean disease on society are higher than natural child delivery. ${ }^{13}$ The maternal mortality rate of cesarean is 2-7 times and the rate of inability is 5-7 times higher than natural child delivery, and finally the emergence of

\author{
Volume 9 Issue 6 - 2018 \\ Fatemeh Setudezadeh, Taraneh \\ Yousefinezhadi \\ Shahid Beheshti University of Medical Sciences, University in \\ Tehran, Iran
}

\begin{abstract}
Correspondence: Taraneh Yousefinezhadi, Safety Promotion and Injury Prevention Research Center, Shahid Beheshti University of Medical Sciences, University in Tehran, Iran, Tel +989123367350, Email t.yousefinezhadi@gmail.com
\end{abstract}

Received: March 08, 2018 | Published: December 26, 2018

maternally vital consequences rate in cesarean with no indications is three times higher than vaginal child delivery.

Studies show in England every one percent increase in cesarean inflicts 5 million pounds cost annually. ${ }^{14}$ A study by World Health Organization has showed 69 countries have more than $15 \%$ cesareans and global saving for its reduction to $15 \%$ is $2 / 32$ billion dollars.

The procedure of performing cesarean in Iran has rapidly increased during 1970 to 2000 so that its rate from $19 / 5 \%$ in 1976 has reached to $48 \%$ in 2010 . Therefore the minimum of cesarean rate in Iran is three times higher than global standards. However, this statistics is related to the information that is recorded in academic hospitals and the statistics of private hospitals is reported to be higher than $90 \%$ of the total of child deliveries. ${ }^{4}$ Meanwhile it is mentioned in the section six of the protocol of our friendly hospitals in comprehensive health system that cesarean rate should be reduced to $15 \%$ in public hospitals and to $20 \%$ in referred hospitals. ${ }^{15,16}$

\section{Main text}

According to the former studies, various factors affect the increase of cesarean. They include factors that affect women's preferences, behavior of health providers and system. The lower rate of natural child delivery tariff in comparison to caesarean delivery and economic efficiency of cesarean in comparison to natural child delivery, ${ }^{17}$ remuneration payment method, ${ }^{9,12}$ the lack of time issue, ${ }^{11}$ the increasing number of young doctors in the field of obstetrics and gynecology who are less familiar with forceps delivery techniques and vacuum, ${ }^{17}$ resident training system, ${ }^{18}$ unpredictability of natural child delivery and high levels of stress resulted from it are the cause of increase in cesarean. ${ }^{5}$

Medical countermeasure is another factor affecting the rate of cesarean. Medical countermeasure is resulted from the effects of law on the obligations of medical profession and fear of law; $;^{11,19}$ the high amount of atonement money, the existence of different centers to deal with medical errors and lack of the existence of a practical guide for judgment are listed in this group. The lack of collaboration between obstetricians and gynecologists and midwives as well as the lack of trust among them and the lack of an appropriate job description definition for service providers is also another reason in reduction 
of the tendency for natural child delivery. Reduction of natural child delivery management by midwives and their training system affects this procedure as well. ${ }^{5}$

Studies show that women's view and their personal preferences can be an important factor in decision making regarding the type of child delivery. ${ }^{20}$ Women's preferences for going under the cesarean operation are under the influence of cultural and social factors and their understanding of safety. ${ }^{12,21,22}$ In compare with natural child deliver, women who go under cesarean operation with no indication have higher education. ${ }^{23}$ It can be said the increase in the level of education, better job opportunity and higher income for individuals and rising of family economic strength cause a higher affordability for paying the costs of cesarean and on the other hand by the increase in individual's socio-economic class and a higher tendency to luxury, cesarean is considered as a type of luxury and a sign of upper class and a higher culture and natural child delivery is specific to people with low socioeconomic class levels and low income which causes the increase in cesarean..$^{19}$ The older age to bear first child,,$^{17,24,25}$ specifying a special date for child delivery, presence of the husband at delivery time, earlier discharge, the mother and the child in the same room, breastfeeding immediately after giving birth, ${ }^{26}$ and former cesarean record ${ }^{2}$ are also other affecting factors in the increase in cesarean rate. Fear of the child delivery pain, ${ }^{11}$ fear of hurting the fetus, and fear of causing disorders in marital relationship ${ }^{27,28}$ are also among the main reasons for women to desire cesarean. ${ }^{17}$ Studies suggest that performance of cesarean department staff has a significant role in women's increasing fear of natural child delivery and choosing of cesarean. ${ }^{29}$ It seems patient's ignorance, deficiency of proper training and lack of creating a true attitude among them are other reasons of the increase in cesarean. ${ }^{1,30}$ Lack of the proper facilities in the pain room also reduces women's desire for natural delivery. ${ }^{5}$ So far, the Ministry of Health and Medical Education has taken measures to reduce unnecessary cesarean. Publication of a protocol in 2003 was among these measures but the protocol lacked details and information expected by clinical guides and couldn't made any change in the increasing procedure of cesarean in the country. ${ }^{12}$ Another significant measure was application of a protocol to make hospitals mother friendly whose main purpose was modifying the workflow, providing mothers with a new model of taking care in order to provide, to maintain and to improve maternal health, and in order to improve the quality of natural child delivery cares, reducing costs, and paying attention to the rights of the mother and the baby to provide a better situation for natural child delivery of mothers. The baby-friendly program, the implementation of which is recommended by the World Health Organization to all of member countries, was started in our country since 1990th and now, while the implementation of mother-friendly hospitals has been on the agenda of the Ministry of Health, experts have expressed their concerns about not implementing the standards of the mother and the baby in baby-friendly hospitals. A remarkable example for these efforts is the national protocols of Ministry of Health, Treatment and Education in 1999, a specific purpose of which was to reduce unnecessary cesareans. These clinical guiding protocols have covered the most prevalent complications of child delivery and for each complication a specific protocol is developed by the experts of the Ministry of Health. Implementation of this protocol couldn't reduce cesarean rate either. ${ }^{14}$

\section{Suggestions}

Conducted researches showed that using of the clinical performance protocols and mother-friendly hospitals does not guarantee reduction of cesarean. ${ }^{14}$ Accordingly the studies that can help reducing cesarean rate are analyzed and based on the available evidences policy options are presented in the form of following table. These options have been classified in the form of four axis factors of social, organizational, economic and governance. ${ }^{31}$

\section{Social factors}

Using public groups including specialist medics, midwives, interested nurses, housewives, teachers, health correspondent, family health personnel and .... For instruction and consultation of pregnant women.

Building trust between physicians, midwives and systems to improve teamwork. ${ }^{5}$

\section{Organizational factors}

a. Encouraging mothers and emotional support of mother in cases of the fear of natural child delivery. ${ }^{32}$

b. The availability of a quiet and serene environment with light colors and provision of a special space for each patient to reduce patient anxiety. ${ }^{6}$

c. Using of group training during pregnancy to increase understanding and changing of women's attitudes in choosing type of child delivery. ${ }^{29}$

d. Giving evidence-based training about natural child delivery to mothers during pregnancy in order to overcome the fear of ambiguities of pregnancy to pregnant women giving birth, stating advantages and disadvantages of each type of delivery. $1,20,25,33-35$

e. Holding relaxation classes during pregnancy. ${ }^{36}$

f. Presence of a trained company while giving birth

g. Making women who are going to have their first giving birth familiar with the delivery room atmosphere, staff and facilities.

h. Expanding the use of physiology deliveries and painless deliveries such as delivery in water. ${ }^{37}$

i. Changing the behavior of service providers specially the physicians by developing inspection and feedback. ${ }^{5}$

j. Using on call physicians in hospitals. ${ }^{5}$

\section{Economic factors}

i. Modification of insurances systems and covering the midwives services in payment system. ${ }^{5}$

ii. Changing payment of the physician's fee into salary and wage for delivery. ${ }^{5}$

\section{Governance factors}

a) Review of academic training of assistants of women and nursing students to change individual's attitudes about the delivery methods. $^{14}$

b) Requiring physicians and medical personnel for enough explanation of the reasons of performing cesarean and its dangers and advantages to mothers. ${ }^{30}$

c) Monitoring and controlling to equalize the tariff of the natural delivery and cesarean. ${ }^{4}$

d) Investigation and monitoring on cesareans with no indications. ${ }^{38}$ 
e) Issuing of performing natural child delivery at homes by midwives. ${ }^{5}$

f) Support of the painless and physiologic deliveries. ${ }^{5}$

g) Specifying the time if transferring the responsibility from midwives to physicians and passing of job description regulations and their economic issues in law. ${ }^{5}$

h) Revision of the standards of allocation of human resources and modification of employment regulations due to lack of midwives in the hospital. ${ }^{5}$

i) Determination of the optimal cesarean rate in the country and in hospitals depending on the level of coverage and population covered services. $^{5}$

j) Expanding the capabilities of midwives by revision and developing of trainings. ${ }^{5}$

k) Improving of legal system to increase

1) Improve the legal system to increase motivation of specialist for natural delivery, the use of a unified and integrated center for dealing with complaints, establishing scientific guidelines for judgment. ${ }^{5}$

m) Mandate the affirmation of the proper cases of cesarean by another physician. ${ }^{4}$

\section{Conclusion}

Regarding the presented suggestions and one of the reasons of cesarean being ignorance of mothers in choosing the type of child delivery, providing mothers with evidence-based training about natural child delivery during pregnancy in order to remove pregnant mother's fears of the unknowns of a delivery, expressing advantages and disadvantages of each type of child delivery can be considered to be of the most important policy options. Many studies were conducted and results have showed a significant statistical difference in awareness and attitudes of women after training in comparison to before training and choosing type of child delivery has also changed in mothers after attending training courses. ${ }^{29,30}$ Presenting evidence-based training to mothers about natural child delivery during pregnancy include in improvement of the quality of life after vaginal delivery, a higher average of mental disorder in the mothers who had cesarean after delivery time period in comparison to natural child delivery, no sexual functionality difference in women who had natural child delivery can have a potential influence in decision making of mothers and it can be suggested as the best policy option.

Another policy option is changing of the behavior of the service providers and developing inspection since physicians are important criteria for mothers and they can be influential in mothers' decision making. The physicians and medical staff can have a considerable role in educating mothers and reducing the rate of cesarean, especially when they decide to have to cesarean delivery with no specific medical indications. ${ }^{30}$ By developing inspection and monitoring on cesarean with no specific indications, increasing the motivation of specialists to perform vaginal delivery, revision of academic training of residents of the women field and students of midwife nursing in order to change individuals' attitudes to the types of child delivery and... getting closer to this objective can be possible. , $^{5,1438}$

\section{Acknowledgments}

None.

\section{Conflicts of interest}

The author declares that they do not have any conflicts of interest.

\section{References}

1. Jamshidi Manesh M, Oskouie S, Jouybary L, et al. The Process of Women's Decision Making for Selection of Cesarean Delivery. Iran Journal of Nursing. 2009;21(56):55-67.

2. Mohammad beigi M, Tabatabaee H, Salehi N, et al. Factors Influencing Cesarean Delivery Method in Shiraz Hospitals. Iran Journal of Nursing. 2009;21(56):37-45.

3. Behmanesh SH, Iranfar SH, Kamravamanesh M, et al. Attitude of pregnant women about delivery Methods. Journal of Kermanshah University of Medical Sciences. 2009;13(1):101-102.

4. Moayed Mohseni S, Sohrabi Z. The predicted growth rate of cesarean been realized? Payesh. 2011;10(2):261-264.

5. Yazdizadeh B, Nedjat S, Mohammad M, et al. Cesarean section rate in Iran, multidimensional approaches for behavioral change of providers: a qualitative study. BMC Health Services Research. 2011;11:159.

6. Askari F, Atarodi A, Torabi SH, et al. Women's labor experience: a phenomenological study. Ofogh-e-Danesh. 2010;16(1):39-47.

7. Shakerian B. Study frequency of cesarean and it causes in Chaharmahal and bakhtiari. Journal of sharkord university of medical sciences. 2004;6(1):63-69.

8. Charoenboon $\mathrm{CH}$, Srisupundit K, Tongsong TH. Rise in cesarean section rate over a 20 -year period in a public sector hospital in northern Thailand. Arch Gynecol Obstet. 2013;287:47-52.

9. Cai W, Marks J, Chen $\mathrm{CH}$, et al. Increased cesarean section rates and emerging patterns of health insurance in Shanghai, China. American Journal of Public Health. 1998;88(5):777-780.

10. Ghaffari M, Sharifirad GH, Akbari Z, et al. Health belief modelbased education \& reduction of cesarean among pregnant women: an interventional study. Health system Research J. 2011;7(2):200-208.

11. Changizi N, Farahani Z. Cesarean section: trend, analysis of the trend and causes. Iranian obstetrician journal. 2013;8(1):9-14.

12. Ostovar R, Rashidian A, Pourreza A, et al. Developing criteria for Cesarean Section using the RAND appropriateness method. BMC Pregnancy and Childbirth. 2010;10:52.

13. Mamak SH, Majlesi F, Azari S. Study cesarean rate and factors influencing on it in Tehran hospitals. Payesh. 2002;3:5-10.

14. Yavangi M, Sohrabi MR, Alishahi Tabriz A. Effect of Iranian ministry of health protocols on cesarean section rate: a quasi- experimental study. $J$ Res Health Sci. 2013;13(1):48-52.

15. Gibbons L, Belizan JM, Lauer JA, et al. Inequities in the use of cesarean section deliveries in the world. Am J Obstet Gynecol. 2012;206:331-332.

16. Laluei A, Kashanizadeh N, Teymouri M. The influence of academic educations on choosing preferable delivery method in obstetrics medical team: investigating their viewpoints. Education in medical sciences journal. 2009;9(1):69-78.

17. Amiri Farahani L, Abbasi Shavazi MJ. Caesarean section change trends in iran and some demographic factors associated with them in the past three 
decades. Journal of Fasa University of Medical Sciences. 2012;2(3):127134.

18. Wu JM, Hundley AF, Visco AG. Elective primary cesarean delivery: attitudes of urogynecology and maternal-fetal medicine specialists. Obstet Gynecol. 2005;105(2):301-306.

19. Arjmandi Rafsanjan B, Farzin Moghaddam S. Assessment of the level of the pregnant womens knowledge towards the advantages and disadvantages of normal vaginal delivery and cesarean section, Tehran. Journal of Iran University of medical sciences. 2005;4(55):13-22.

20. Baghdari N, Khosravi Z, Mazloom R, et al. Comparison of sexual function in women after vaginal delivery and cesarean in health centers of Mashhad. IJOGI. 2012;15(30):8-14.

21. McCourt CH, Weaver J, Statham H, et al. Elective cesarean section and decision making: a critical review of the literature. Birth. 2007;34(1):6579 .

22. World health organization. Rising caesarean deliveries in Latin America: how best to monitor rates and risks. 2009.

23. World health organization. Caesarean section without medical indication increases risk of short-term adverse outcomes for mothers. 2009.

24. Stafford RS. Alternative strategies for controlling rising cesarean section rates. JAMA. 1990;263(5):683-687.

25. Ranae F. Reasons for Choosing Cesarean among Women Referred to Bea'sat Hospital of Sanandaj. 2004;10(3):277-286.

26. Khakbazan Z, Babai GH, Jamshidi F, et al. Study reasons for choosing cesarean section by maternal in health centers of Rasht. Hayat. 2004;10(22):51-60.

27. Nuttall CH. The caesarean culture of Brazil. BMJ. 2000;320:1080-1083.

28. Quadros LG. Caesarean section controversy. Brazilian obstetricians are pressured to perform caesarean sections. BMJ. 2000;320(7241):1073.
29. Tofighi Niaki M, Behmanesh F, Mashmuli F, et al. The Effect of Prenatal Group Education on Knowledge, Attitude and Selection of Delivery Type in Primiparous Women. Journal of education in medical sciences. 2010;10(2):124-130.

30. Mohammaditabar SH, Kiani A, Heidari M. The Survey on Tendencies of Primiparous Women for Selecting the Mode of Delivery. J Babol Univ Med Sci. 2009;11(3):54-59.

31. Ganji F, Reisi R, Khosravi S, et al. Effect of a participatory intervention to reduce the number of unnecessary cesarean sections performed in Shahrekord, Iran. J Shahrekord Univ Med Sci. 2006;8(1):14-18.

32. Yassaee F, Mohseni B. Role of mother's preference for the type of Pejouhesh. 2007;31(2):129-132.

33. Nikpour M, Abedian Z, Mokhber N, et al. Comparison of quality of life in women after vaginal delivery and cesarean section. Journal of Babol University of Medical Sciences. 2011;13(1):44-50.

34. Khosravi M, Armat MR, Khadem N. Cesarean rate and its related factors in. Journal of Nursing and Midwifery.2007; 17(58):21-27.

35. Mangoli M, Zare Mobini F, Ramezani T. Impact of delivery method on postpartum mental health. Journal of Hormozgan University of Medical Sciences. 2011;14(4):326-334.

36. Azizi M, Salari P. C-section in request: an ethical approach. Journal of Medical Ethics and History of Medicine. 2009;2(2):55-66.

37. Akbari S, Rashidi N, Changavi F, et al. The effect of water birth on the duration of labor and pain level in comparision with land birth. Yafteh. 2008;10(3):39-46.

38. Souza JP, Gülmezoglu AM, Lumbiganon P, et al. Caesarean section without medical indication increases risk of short-term adverse outcomes for mothers: the 2004-2008 WHO Global Survey on Maternal and prenatal Health. BMC Med. 2010;8:71. 